Article

\title{
Improving the Substrate Affinity and Catalytic Efficiency of $\beta$-Glucosidase Bg13A from Talaromyces leycettanus JCM12802 by Rational Design
}

\author{
Wei Xia ${ }^{1,+} \mathbb{D}$, Yingguo Bai ${ }^{2, *}$ and Pengjun Shi ${ }^{1, *}$ \\ 1 Institute of Food Science and Technology, Chinese Academy of Agricultural Sciences, Beijing 100081, China; \\ weixia@jiangnan.edu.cn \\ 2 Institute of Animal Science, Chinese Academy of Agricultural Sciences, Beijing 100193, China \\ * Correspondence: baiyingguo@caas.cn (Y.B.); shipengjun@caas.cn (P.S.) \\ + Current Address: State Key Laboratory of Food Science and Technology, Jiangnan University, \\ 1800 Lihu Ave-nue, Wuxi 214122, China.
}

Citation: Xia, W.; Bai, Y.; Shi, P. Improving the Substrate Affinity and Catalytic Efficiency of $\beta$-Glucosidase Bgl3A from Talaromyces leycettanus JCM12802 by Rational Design. Biomolecules 2021, 11, 1882. https:// doi.org/10.3390/biom11121882

Academic Editors: Eric Sorin and Mark Harrison

Received: 20 October 2021

Accepted: 9 December 2021

Published: 15 December 2021

Publisher's Note: MDPI stays neutral with regard to jurisdictional claims in published maps and institutional affiliations.

Copyright: (C) 2021 by the authors. Licensee MDPI, Basel, Switzerland. This article is an open access article distributed under the terms and conditions of the Creative Commons Attribution (CC BY) license (https:/ / creativecommons.org/licenses/by/ $4.0 /)$.

\begin{abstract}
Improving the substrate affinity and catalytic efficiency of $\beta$-glucosidase is necessary for better performance in the enzymatic saccharification of cellulosic biomass because of its ability to prevent cellobiose inhibition on cellulases. Bgl3A from Talaromyces leycettanus JCM12802, identified in our previous work, was considered a suitable candidate enzyme for efficient cellulose saccharification with higher catalytic efficiency on the natural substrate cellobiose compared with other $\beta$-glucosidase but showed insufficient substrate affinity. In this work, hydrophobic stacking interaction and hydrogen-bonding networks in the active center of Bgl3A were analyzed and rationally designed to strengthen substrate binding. Three vital residues, Met36, Phe66, and Glu168, which were supposed to influence substrate binding by stabilizing adjacent binding site, were chosen for mutagenesis. The results indicated that strengthening the hydrophobic interaction between stacking aromatic residue and the substrate, and stabilizing the hydrogen-bonding networks in the binding pocket could contribute to the stabilized substrate combination. Four dominant mutants, M36E, M36N, F66Y, and E168Q with significantly lower $K_{\mathrm{m}}$ values and 1.4-2.3-fold catalytic efficiencies, were obtained. These findings may provide a valuable reference for the design of other $\beta$-glucosidases and even glycoside hydrolases.
\end{abstract}

Keywords: $\beta$-glucosidase; cellobiose; enzyme engineering; substrate affinity; molecular dynamics simulation

\section{Introduction}

Cellulose is the most abundant polysaccharide in nature and constitutes the highest proportion of municipal and agricultural wastes. Additionally, it represents the most valuable source of renewable energy and raw materials [1]. The worldwide consensus is that the efficient utilization of cellulosic agricultural wastes is critical in generating sustainable biofuel production methods [2]. The enzymatic degradation of cellulose to glucose is generally accomplished by a synergic action of three kinds of glycoside hydrolases, such as endo$\beta$-glucanase (EG, EC 3.2.1.4), cellobiohydrolase (CBHs, EC 3.2.1.91), and $\beta$-glucosidase (BGLs, EC 3.2.1.21) [3,4]. Fungi, as the primary producers of cellulose-degrading enzymes, have received most of the attention regarding biotechnological applications. However, as previously reported, the lack of endogenous $\beta$-glucosidase is always the fatal defect of current industrial microorganisms used for the enzymatic degradation of cellulose, such as Trichoderma reesei $[5,6]$. This is because the end-product inhibition of CBHs by cellobiose, which is the natural substrate of $\beta$-glucosidase, can seriously reduce the overall conversion rate of cellulose into glucose $[7,8]$. Therefore, $\beta$-glucosidases with high qualities are essential for enhancing the utilization efficiency of cellulosic agricultural wastes $[9,10]$. 
$\beta$-glucosidases, mainly catalyzing the hydrolysis of the $\beta$-1,4-glycosidic linkage in various disaccharides, oligosaccharides, and alkyl- and aryl- $\beta$-D-glucosides, have been classified into GH families 1, 3, 5, 9, 30, and 116 based on their amino acid sequences [11-13]. The different $\beta$-glucosidases vary significantly regarding enzymatic properties, such as reaction optimums, substrate specificities, catalytic efficiencies, tolerance to unfavorable conditions, and inhibition constants for glucose [8]. Most of the microbial $\beta$-glucosidases employed today in cellulose hydrolysis belong to GH family $3[11,14]$. Increasing research has focused on the protein structure, ligand-binding mode, and critical residues of GH3 $\beta$-glucosidases. Structural analysis has revealed three conserved substrate recognizing residues for the subsite +1 of cellobiose in the $\beta$-glucosidases capable of hydrolyzing cellobiose, for instance, Trp68, Phe305, and Tyr511 of AaBGL1 from Aspergillus aculeatus (PDB 4IIB) [15], and Trp37, Phe260, and Tyr443 of HjCel3A from Hypocrea jecorina (PDB 3ZYZ) [16]. Moreover, several recent studies have reported that several critical residues at the entrance or inside the catalytic pocket could influence the enzymatic properties of $\beta$ glucosidases. These include the Ile substitution of conserved Trp at the pocket entrance that makes HiBgl3B from Humicola insolens Y1 be a strict aryl- $\beta$-glucosidase [11]. Furthermore, three amino acid changes contribute markedly to the thermostability of $\beta$-glucosidase BglC from Thermobifida fusca [17], and R97 and Y331 could modulate the optimum $\mathrm{pH}$ of GH1 $\beta$-glycosidase from Spodoptera frugiperda [18].

Significant efforts have been made to improve the enzymatic properties of the existing GH3 enzymes owing to the practical benefit of $\beta$-glucosidases in the biofuel industry. A series of valuable promotions have been achieved by newly developed structure-based rational design, which was assisted by computational algorithms in the enzyme engineering of GH3 $\beta$-glucosidases [19]. Beneficially combined mutants with increased hydrolytic activity for synthetic and natural substrates have been obtained by directed evolution of a fungal GH3 $\beta$-glucosidase BGL1 in Saccharomyces cerevisiae [20]. The Q201E mutant of AaBGL1, generated by site-saturation mutagenesis, was found to have 2.7-times higher $k_{\text {cat }} / K_{\mathrm{m}}$ toward cellobiose than the WT enzyme [21]. Substitution of Trp512 in $\beta$-glucosidase from Clavibacter michiganensis has been shown to transform the regioselectivity for hydrolyzing gypenoside XVII [22]. Furthermore, the catalytic efficiency in quercetin-4'-glucoside hydrolysis of Thermotoga maritima $\beta$-glucosidase A was enhanced by site-directed mutagenesis [23]. However, most studies were conducted using $p$ NPG as the representative substrate molecule, and few modifications were made on the substrate affinity and catalytic efficiency of GH3 $\beta$-glucosidases toward their natural substrate cellobiose. To improve the practical economic benefits of the enzymatic saccharifications of cellulosic biomass and the subsequent bioethanol production, more attention must be paid to the catalytic performances of $\beta$-glucosidases on cellobiose [9]. Therefore, developing $\beta$-glucosidases with reasonable substrate specificity and high catalytic efficiency is of great importance for the biotransformation of rare ginsenosides.

Our previous work identified and characterized the highly efficient GH3 $\beta$-glucosidase (Bg13A) from Talaromyces leycettanus JCM12802. This had excellent application prospects with a relatively high specific activity and catalytic efficiency of $905 \mathrm{U} / \mathrm{mg}$ and $9096 / \mathrm{s} / \mathrm{mM}$ on $p$ NPG, respectively [14]. Its high catalytic efficiency benefited from the high $k_{\text {cat }}$ value. However, the specific activity and catalytic efficiency of Bgl3A on cellobiose were much lower $(265.5 \mathrm{U} / \mathrm{mg}$ and $75.8 / \mathrm{s} / \mathrm{mM})$ because of its low affinity towards cellobiose. The $\mathrm{K}_{\mathrm{m}}$ value of Bgl3A on cellobiose was $10.4 \mathrm{mM}$, a 57 -fold higher value compared with $p$ NPG. It indicated that, although the $k_{\text {cat }} / K_{\mathrm{m}}$ value of $75.8 / \mathrm{s} / \mathrm{mM}$ was at a relatively high level among GH3 $\beta$-glucosidases, Bgl3A could not work well when the concentration of cellobiose was not very high. Therefore, the low affinity towards cellobiose was a significant bottleneck for practical use of Bgl3A in the biofuel industry, considering that cellobiose was the primary natural substrate of $\beta$-glucosidases in the process of cellulose saccharification. The purpose of this study was to improve the substrate affinity of Bgl3A towards cellobiose by enzyme engineering. Using the site-directed saturation mutagenesis 
method, two improved mutants with 2.3-fold higher catalytic efficiencies on cellobiose were obtained.

\section{Materials and Methods}

\subsection{Strains, Plasmids, and Materials}

Recombinant plasmid pPIC9 harboring the coding sequence bgl3A (gene bank accession KU363626), constructed in our previous work [14], was used as the template for site-saturation mutagenesis. Pichia pastoris strain GS115 (Invitrogen, Carlsbad, CA, USA) was employed as a heterologous expression host for protein preparation. The substrates 4-nitrophenyl $\beta$-D-glucopyranoside ( $p N P G)$, cellobiose, gentibiose, and salicin were purchased from Sigma-Aldrich (St. Louis, MO, USA). LA Taq DNA polymerase, restriction endonucleases, and DNA purification kit were purchased from TaKaRa (Otsu, Japan). DNA ligase and total RNA isolation system kit were purchased from Promega (Madison, WI, USA). All other chemicals were of analytic grade and commercially available.

\subsection{Sequence Analysis, Homology Modeling, and Docking}

The multiple sequence alignment of Bgl3A and other typical GH3 $\beta$-glucosidases was performed using ClustalX version 2.1, followed by a rendering of sequence similarities and secondary structure information in the aligned sequences by the online tool ESPript (http: / / espript.ibcp.fr/ESPript/ESPript/) (accessed on 3 October 2021). The evolutionary conservation analysis was performed using the Weblogo program (http:/ / weblogo. berkeley.edu/logo.cgi) (accessed on 9 December 2021). The homology model of the threedimensional structure of wild-type Bgl3A was homology modeled by the Swiss Model server (https: / / www.swissmodel.expasy.org /) (accessed on 9 December 2021) with the crystal structure of $H$ jCel3A from $H$. jecorina (PDB: 3ZYZ; with 73\% identity) as the template. The obtained optimal model of Bgl3A was evaluated using Verify3D and PROCHECK programs (Supplemental Figure S1). The pairwise cation- $\pi$ interactions were predicted using the online tool PIC (Protein Interactions Calculator, http:/ / pic.mbu.iisc.ernet.in/job.html) (accessed on 9 December 2021), and the evaluation standard required that the distance between the basic and aromatic amino acid was within $6 \AA$ and the angle was appropriate [24].

The structure file of the ligand cellobiose, a representative of the disaccharide substrate of $\beta$-glucosidase, was generated and energy-minimized using the ChemBioOffice software (Version 14.0, CambridgeSoft Corporation, Cambridge, MA, USA). The docking of modeled Bgl3A and cellobiose was carried out by the Autodock4.2 program following the user guide. The docking grids were set as $40 \times 40 \times 40$ with a grid spacing of $1 \AA$ and centered on the $\beta$-carbon atom of the catalyst Glu234. The exhaustiveness for the docking poses was 50. Finally, a suitable enzyme-substrate complex Bgl3A-cellobiose was obtained using binding energy values as the evaluation criterion. The complex structure was further minimized and optimized with a 500-step molecular dynamics (MD) simulation using the Amber 18 package. To avoid the possible errors, we did energy minimization for the model before docking and MD simulation, respectively. Consequently, the binding mode of the substrate cellobiose in the obtained docking model is close to that in the template structure $3 \mathrm{ZYZ}$, indicating that the docking results are reasonable to some extent. Visualization, analysis, and figure preparation of the protein and complex structures were performed with PyMOL (version 1.8.x, Delano Scientific LLC, Berkeley, CA, USA).

\subsection{Identification of Mutagenesis Sites and Mutant Construction}

Based on the structure and docking analysis, three residues (Trp35, Arg65, and Arg167) were proposed to be of great importance for binding the glycoside substrates. However, these binding residues were almost absolutely conservative in GH3 $\beta$-glucosidases. Therefore, three adjacent residues (Met36, Phe66, and Gln168), which showed less conservation and interacted with Trp35, Arg65, and Arg167, respectively, were chosen for mutagenesis to improve the catalytic properties of Bgl3A. Overlap PCR was performed to obtain gene 
fragments of mutated coding sequences using specific primers shown in Supplemental Table S1. The PCR products and PPIC9 vector were both digested by EcoR I and Not I and ligated by T4 ligase to construct the expression plasmids. Sequencing was carried out to verify the coding genes and successful recombination into the pPIC9 vector. The Vector NTI 11.5 software (Invitrogen, Carlsbad, CA, USA) was used to analyze the sequencing results of mutagenesis.

\subsection{Enzyme Expression and Purification}

All recombinant plasmids were digested by the single restriction enzyme $B g l$ II and the completely linearized product was transformed into P. pastoris GS115 competent cells by electroporation using a Gene Pulser X cell Electroporation System (Bio-Rad, Hercules, CA, USA). The cells were coated onto minimal dextrose medium plates and cultured at $30{ }^{\circ} \mathrm{C}$ for 2 days. Then, the screening of transformants with the highest enzyme activity was performed in a $10 \mathrm{~mL}$ tube using the method described in our previous work [14]. For shake-flask fermentation, the recombinant strains were activated in the YPD medium, followed by 2 days of propagation culture in BMGY medium (400 mL), and 2 days of induction culture in BMMY medium $(200 \mathrm{~mL})$ containing $1 \%$ methanol at $30{ }^{\circ} \mathrm{C}$. The cultures were centrifuged $\left(12,000 \times g, 4{ }^{\circ} \mathrm{C}\right.$, and $\left.10 \mathrm{~min}\right)$, and the supernatant was collected and concentrated by a $5 \mathrm{kDa}$ cutoff tangential flow Vivaflow ultrafiltration membrane (Vivascience, Hannover, Germany). Then, the concentrated crude enzymes were desalted by dialysis and purified using anion exchange chromatography (HiTrapQ Sepharose XL, $5 \mathrm{~mL}$ column, $20 \mathrm{mM}$ Tris- $\mathrm{HCl}$ buffer, $\mathrm{pH}$ 8.0). Purified enzymes were subjected to sodium dodecyl sulfate-polyacrylamide gel electrophoresis (SDS-PAGE). Lastly, protein concentration measurements were obtained using the Bradford method with bovine serine albumin (BSA) as the standard.

\subsection{Enzymatic Assays and Kinetic Parameters}

The optimum temperature and $\mathrm{pH}$ of wild-type Bgl3A and all the mutants were determined using $p$ NPG as a substrate. One unit of $\beta$-glucosidase activity was defined as the amount of enzyme that released $1 \mu \mathrm{mol}$ of glucose per minute. For the substrate $p \mathrm{NPG}$, the standard reaction system, containing the appropriately diluted enzyme $(250 \mu \mathrm{L})$ and $\mathrm{Na}_{2} \mathrm{HPO}_{4}$-citric acid buffer of different $\mathrm{pH}$ values containing $2 \mathrm{mM} p \mathrm{NPG}(250 \mu \mathrm{L}$; $50 \mathrm{mM}$ ), was incubated at appropriate temperature for $10 \mathrm{~min}$. Then $1.5 \mathrm{~mL}$ of $1.0 \mathrm{M}$ $\mathrm{Na}_{2} \mathrm{CO}_{3}$ solution was added to terminate the reaction. The amount of $p$-nitrophenol $(p \mathrm{NP})$ released was determined spectrophotometrically by reading the absorbance at $405 \mathrm{~nm}$.

The specific activities towards substrates cellobiose and gentiobiose were assayed using the glucose oxidase-peroxidase (GOD-POD) method under the respective optimal conditions. The standard reaction systems consisted of an appropriately diluted enzyme $(70 \mu \mathrm{L})$ and substrate solution $(70 \mu \mathrm{L})$ with a concentration of $2 \mathrm{mM}$ in $50 \mathrm{mM} \mathrm{pH} 4.5$ $\mathrm{Na}_{2} \mathrm{HPO}_{4}$-citric acid buffer. After a 10-min incubation at appropriate temperature, the reactions were terminated using a boiling water bath. GOD-POD coloring solution $(2.1 \mathrm{~mL})$ was then added into the system, and the absorbances at $520 \mathrm{~nm}$ were measured to determine the amount of released glucose. Each experiment was performed in triplicate.

The kinetic parameters, Michaelis constant $\left(K_{\mathrm{m}}\right)$ and kinetic constant $\left(k_{\text {cat }}\right)$, were determined under the respective optimal conditions of wild-type Bgl3A and all mutants for $5 \mathrm{~min}$ in $\mathrm{Na}_{2} \mathrm{HPO}_{4}$-citric acid buffer $(100 \mathrm{mM})$ containing 1-20 mM of different substrates. The data were plotted and fitted by Graphpad 6.0 software (GraphPad Software, San Diego, CA, USA) to calculate the kinetic parameters.

\subsection{Simulation and Calculation of Binding Energy}

MD simulations were carried out with the AMBER 18 simulation packages according to the instructions of the reference manual $[25,26]$. The topologies and parameters of the enzyme and substrate cellobiose in the complex structure were generated by the Amber ff14SB [27] and GLYCAM06-1 force field, respectively. The SHAKE algorithm was 
employed during the MD simulation process to constrain all bonds relating to hydrogen atoms, and the time step was set as $0.002 \mathrm{ps}$. The simulation system was immersed in a cubic TIP3P water box with a boundary distance of $1.0 \mathrm{~nm}$ for each protein. After, appropriate sodium ions were added to neutralize the negative charge to ensure that the whole system remained electrically neutral. The cutoff radius of the non-bonding interaction was set to $12 \AA$, and the Particle Mesh Ewald (PME) method was used to deal with the long-range electrostatic interaction. The energy minimization simulation included equilibrium of solvent molecules for $2 \mathrm{ps}$ and minimization of the whole system for $50 \mathrm{ps}$. The system was slowly heated from $0 \mathrm{~K}$ to $300 \mathrm{~K}$ under the control of the Langevin algorithm and subsequently equilibrated for 500 ps at 1.0 atmospheric pressure to ensure that the water density reached $1.0 \mathrm{~g} / \mathrm{cm}^{3}$. Finally, a $100 \mathrm{~ns}$ production MD simulation was run under constant pressure, and the coordinate trajectory was recorded every $10 \mathrm{ps}$. The program, cpptraj, was employed to analyze the generated trajectory files [28].

To calculate the binding free energy, 2000 snapshots with equal intervals were extracted from the 20-100 ns production MD simulation trajectory. Energy analyses were performed using the molecular mechanics/Poisson-Boltzmann surface area (MM/PBSA) method. In this method, the total energy of a solvated molecule could be cataloged into three major items and had the following quantitative relationship Equation (1) [29].

$$
G=E_{\mathrm{bnd}}+E_{\mathrm{el}}+E_{\mathrm{vdW}}+G_{\mathrm{pol}}+G_{\mathrm{np}}-T S
$$

$E_{\mathrm{bnd}}, E_{\mathrm{el}}$ and $E_{\mathrm{vdW}}$ represented the standard MM energy terms from bonded (bond, angle, and dihedral), electrostatic and van der Waals interactions. And $G_{\mathrm{pol}}$ and $G_{\mathrm{np}}$ are the polar and non-polar contributions to the solvation free energies, respectively. TS was the energy component involving the entropy effect. However, $\Delta S$ was not calculated in this study because the substrates in all complexes were identical and therefore $\Delta S$ would not significantly impact the results. The difference value between $G_{\text {complex }}$ and $\left(G_{\text {receptor }}+G_{\text {ligand }}\right)$ was considered the change in Gibbs free energy caused by the binding process, which is approximately equal to the binding energy of the receptor-ligand complex. Thus, the binding free energy ( $G_{\text {binding }}$ ) was calculated according to the following formula Equation (2):

$$
G_{\text {binding }}=\Delta E_{\mathrm{el}}+\Delta E_{\mathrm{vdW}}+\Delta G_{\mathrm{pol}}+\Delta G_{\mathrm{np}}
$$

\section{Results and Discussion}

\subsection{Substrate Binding Analysis}

According to our previous study, poor substrate affinity was the main reason for the low catalytic efficiency of Bgl3A towards natural glycoside substrates. Increasing the enzyme's substrate affinity was the most direct strategy for improving its catalytic efficiency. To analyze the enzyme's binding mode, a credible complex structure of Bgl3A and cellobiose was obtained by homologous modeling and molecular docking (evaluation information shown in Supplemental Figure S1). The substrate-binding pocket of Bgl3A was located in a cavity on the inner side of the protein (Figure 1A). Several binding residues were distributed in five flexible loops around the pocket, and they formed interactions with the substrate molecule, resulting in them playing a vital role in catalysis (Figure 1B).

Two major amino acid groups contributed to the substrate binding. The first group was the hydrophobic region formed by three hydrophobic amino acid residues, Met36, Trp35, and Phe258, which served as the hydrophobic stacking point for the +1 subsite of cellobiose. Among them, the hydrophobic interaction formed between Trp35 and C3/C4 atoms of the substrate +1 subsite was the primary hydrophobic interaction and undertook the role of capturing substrate molecules from the solvent (Figure 2A). Several published structural research studies have drawn a similar conclusion, and demonstrated that Trp68, Phe305, and Tyr511 of AaBGL1 (PDB 4IIB) from Aspergillus aculeatus and Trp37, Phe260, and Tyr443 of HjCel3A (PDB 3ZYZ) from Hypocrea jecorina were three conserved residues of great importance for recognizing cellobiose $[15,16]$. Moreover, the substitution of conserved 
W Trp48 with Ile at the pocket entrance made HiBgl3B from Humicola insolens Y1 to be a strict aryl- $\beta$-glucosidase, inhibiting its activity towards all disaccharides [11]. 
A

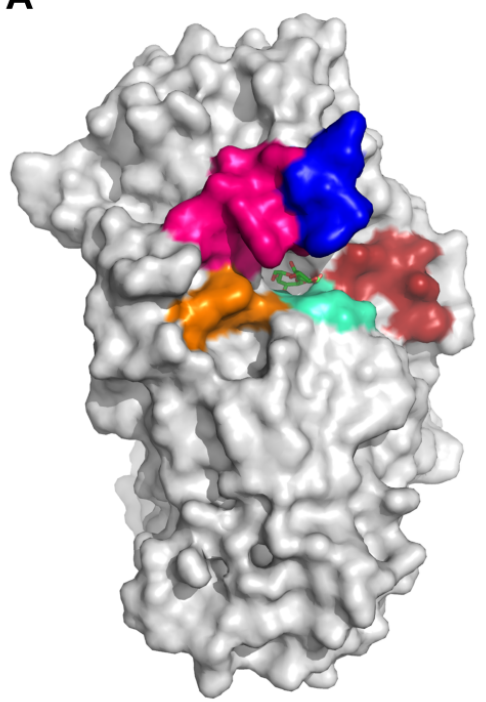

B

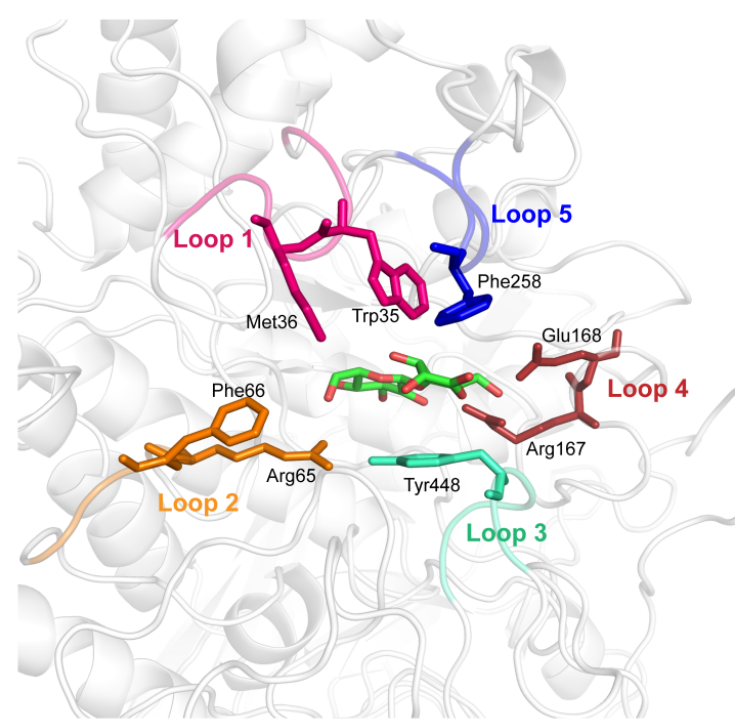

Figure 1. Docking analyses of Bgl3A. Stereoview of modeled Bgl3A and the substrate-binding pocket depicted as surface (A) and cartoons and sticks (B). Substrate binding sites and five flexible loops surrounding the pocket are indicated.

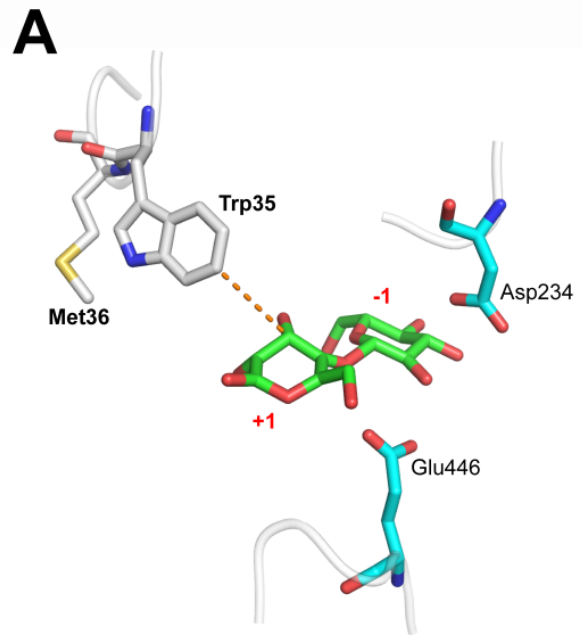

B

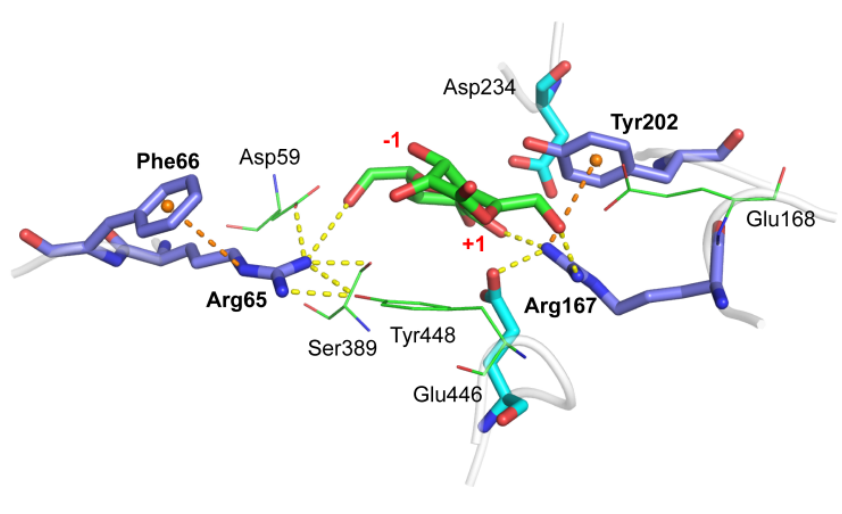

Figure 2. Substrate-binding interactions. Detailed interactions between substrate cellobiose and the hydrophobic Trp35 (A) and hydrogen bond-forming Arg65 and Arg167 (B). The hydrophobic interaction and the cation- $\pi$ interactions are shown in dotted brown lines, and the hydrogen bonds are shown in dotted yellow lines.

Another group involved in the substrate binding was the polar residues, which formed a hydrogen bond network with the substrate and within themselves. This hydrogen bond network was centered on Arg65 and Arg167 and promoted the formation of the correct substrate conformation and catalytic residues. Further analysis of polar interactions showed that the terminal amine $\mathrm{NH}_{2}$ of Arg65 formed a hydrogen bond with the $\mathrm{C} 6-\mathrm{OH}$ in the -1 subsite and interacted broadly with other substrate binding sites such as Asp59, Ser389, and Tyr448. Similarly, Arg167 formed hydrogen bonds with the C6-OH in the +1 subsite, the $\mathrm{C} 2-\mathrm{OH}$ in the -1 subsite, and the $\mathrm{OE} 2$ atom of the nucleophilic catalytic residue Glu446 (Figure 2B). The locations and spatial dynamics of these two residues determined the stability and strength of the hydrogen bond network in the binding pocket. Some computational studies also emphasized the importance of these two conserved arginines, which reported that $\operatorname{Arg} 169$ and Arg67 stabilized the glucose at the acceptor site (subsite +1 ). Additionally, disrupting the hydrogen bond networks reduced the affinity and reactivity of a sugar acceptor $[30,31]$. 


\subsection{Mutant Design}

Enhancing or stabilizing the binding capacities of these two interaction components was a feasible way to improve the substrate affinity of the enzyme. However, multiple sequence alignment showed that the residues Trp35, Arg65, and Arg167 were almost absolutely conserved in the genetic evolution of GH3 $\beta$-glucosidases, which illustrated their importance laterally (Figure 3). As alternatives, three adjacent residues which showed no sequence conservation (Figure 4) were supposed to affect substrate binding by interacting with the previously mentioned three conserved binding sites. We observed that position 36 was close to Trp35 in space, and this might be Gln, Glu, Asp, Asn, and Gly as an alternative to Met in other GH3 $\beta$-glucosidases. Therefore, Met36 was chosen as the site-saturation mutagenesis site for catalytic efficiency improvement in Bgl3A. Moreover, aromatic residues Phe 66 and Tyr202 were predicted to form pairwise cation- $\pi$ interactions with the alkaline residues Arg65 and Arg167, respectively. The cation- $\pi$ interaction is a noncovalent interaction formed between positive cations or groups and benzene rings.
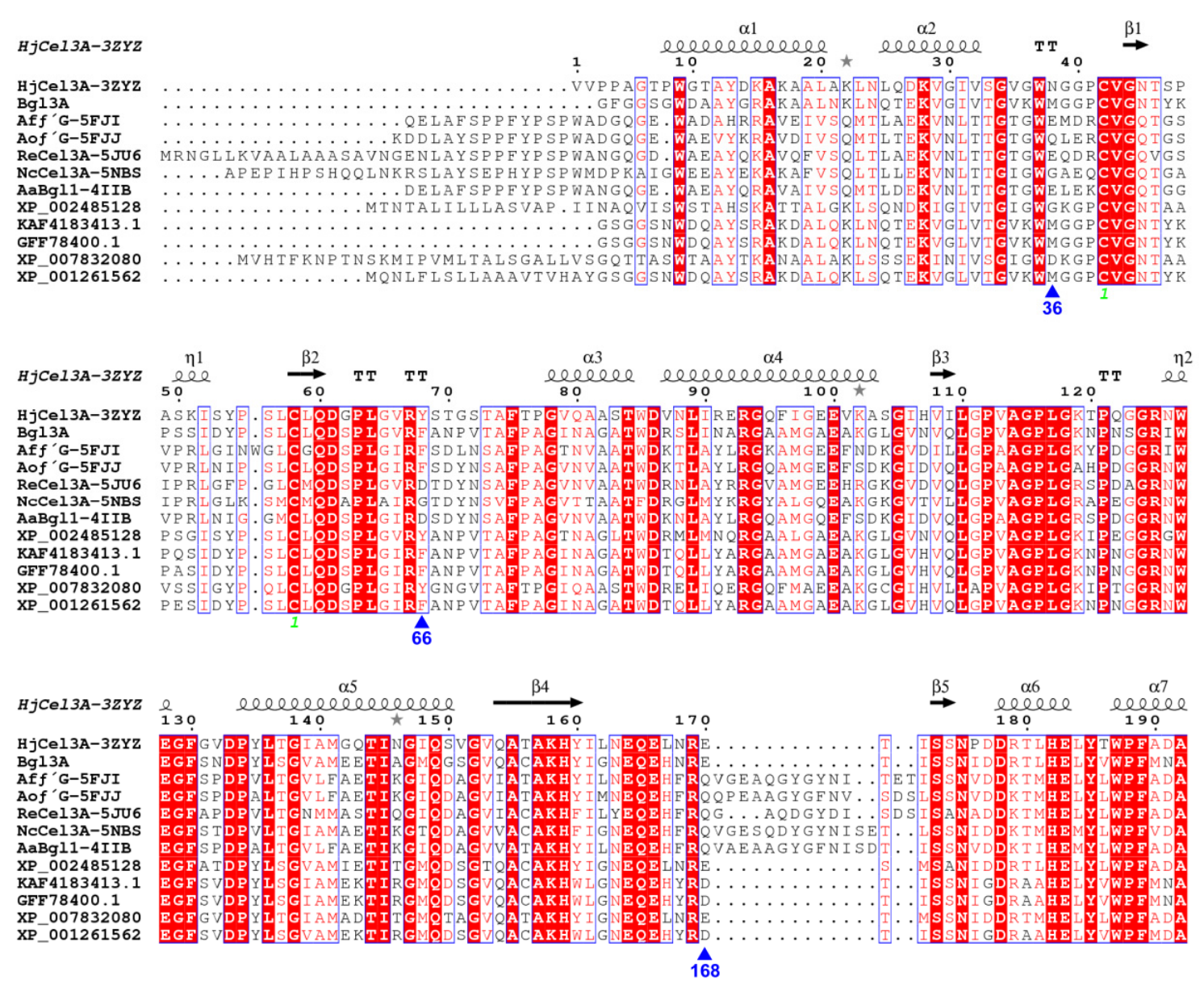

Figure 3. Multiple sequence alignment of Bgl3A with other GH3 $\beta$-glucosidases, namely, HjCel3A from Hypocrea jecorina (PDB: 3ZYZ) [15], Af $\beta$ G from Aspergillus fumigatus (PDB: 5FJI) and Ao $\beta \mathrm{G}$ from Aspergillus oryzae (PDB: 5FJJ) [32], ReCel3A from Rasamsonia emersonii (PDB: 5JU6) [33], NcCel3A from Neurospora crassa OR74A (PDB: 5NBS) [34], AaBgl from Aspergillus aculeatus (PDB: 4IIB) [16]. The multiple sequence alignment was performed using ClustalX version 2.1, and rendered by the online tool ESPript (http:/ / espript.ibcp.fr/ESPript/ESPript/). Strictly identical amino acids were showed in red background color and white characters, and groups of similar amino acids were showed in red characters. Blue frames represented similarity across groups. 

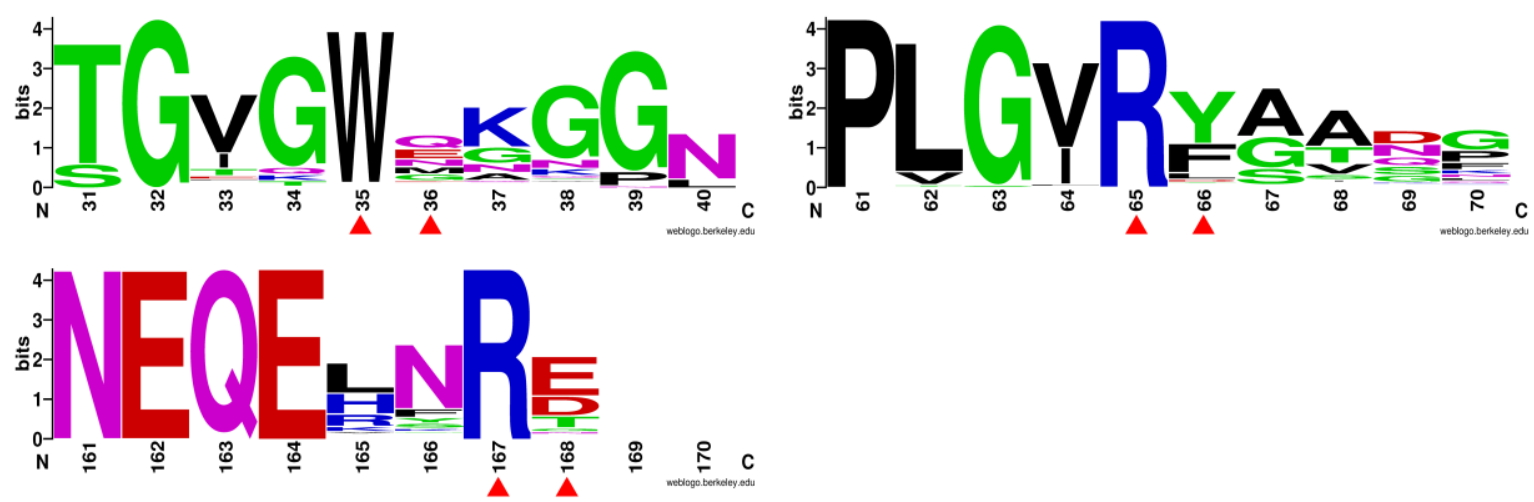

Figure 4. Evolutionary conservation analyses of residues Trp35/Met36, Arg65/Phe66, and Arg167/Glu168. The evolutionary conservation analysis was performed using the Weblogo program (http:/ / weblogo.berkeley.edu/logo.cgi).

Many studies have documented that the cation- $\pi$ interaction widely exists in protein structures and enhances the conformational stability of the bonded residues, where Lys or Arg side chains interact with Phe, Tyr, or Trp [35,36]. As with other noncovalent aromatic interactions in protein structure, the cation- $\pi$ interaction includes a substantial electrostatic component [37] and can be enhanced by increasing the electronegativity of the aromatic rings. Glu168, a negatively charged and genetically variable residue adjacent to Arg167 (Figure 2B), was considered to interfere with the cation- $\pi$ interaction between Arg65 and Phe66. In summary, site-saturation mutagenesis at position 36 and site-directed mutagenesis of F66Y and E168Q were designed to improve the substrate affinity and catalytic efficiency of the enzyme Bgl3A.

\subsection{Catalytic Performances of Wild-Type Bgl3A and Its Mutants}

Specific activities and kinetic parameters towards $p$ NPG and cellobiose of wild-type and all mutant enzymes were determined under respective optimum conditions $\left(75^{\circ} \mathrm{C}\right.$ for wild type and most mutant enzymes, and $70^{\circ} \mathrm{C}$ for M36V/S/C/K/D). For the artificial substrate $p$ NPG, nearly all mutants decreased catalytic efficiencies because of the significant increase in $K_{\mathrm{m}}$ values (Table 1 ). This may be because our design targets mainly glycosylbased substrates. Among mutants at position 36, M36E and M36N exhibited 2.3-fold higher catalytic efficiencies on cellobiose with higher $k_{\text {cat }}$ values and much lower $K_{\mathrm{m}}$ values compared with wild-type Bgl3A. Furthermore, F66Y and E168Q also showed $60 \%$ and $40 \%$ improvement, respectively, in catalytic efficiencies on cellobiose because of the substantially decreased values of $K_{\mathrm{m}}$, although their $k_{\text {cat }}$ values were slightly lower than the wild-type (Table 1). The $K_{\mathrm{m}}$ values of four dominant mutants, M36E (5.2 mM), M36N (4.76 mM), F66Y (4.3 mM), and E168Q (5.0 mM), were approximately halved. This indicated that better substrate affinities with natural glycoside substrates such as cellobiose were obtained by engineering the adjacent residues of conserved binding sites. Further determinations of enzyme performance on gentiobiose confirmed that both mutants M36E and M36N showed certain degrees of improvement in catalytic efficiencies on gentiobiose with lower $K_{\mathrm{m}}$ values of $2.9 \mathrm{mM}$ and $3.1 \mathrm{mM}$, respectively, compared with $5.4 \mathrm{mM}$ for wild-type Bgl3A (Table S2). 
Table 1. Reaction optimum, specific activities, and kinetic parameters of wild-type Bgl3A and its mutants towards $p$ NPG and cellobiose.

\begin{tabular}{|c|c|c|c|c|c|c|c|c|c|c|c|c|}
\hline \multirow[b]{2}{*}{ Enzymes } & \multicolumn{2}{|c|}{ Optima } & \multicolumn{4}{|c|}{$p$ NPG } & \multicolumn{4}{|c|}{ Cellobiose } & \multirow[b]{2}{*}{$\begin{array}{c}\text { Fold Change } \\
\text { in } k_{\text {cat }} / K_{\mathrm{m}} \text { on } \\
\text { Cellobiose }\end{array}$} & \multirow[b]{2}{*}{$\begin{array}{c}\text { Activity } \\
\text { Ratio } \\
\text { Cellobiose/pNPG }\end{array}$} \\
\hline & $\mathrm{T}\left({ }^{\circ} \mathrm{C}\right)$ & $\mathrm{pH}$ & $\begin{array}{l}\text { Specific } \\
\text { actvitity } \\
\left(\mathrm{U} \cdot \mathrm{mg}^{-1}\right)\end{array}$ & $K_{\mathrm{m}}(\mathrm{mM})$ & $\begin{array}{l}k_{\text {cat }} \\
\left(\mathrm{s}^{-1}\right)\end{array}$ & $\begin{array}{c}k_{\text {cat }} / K_{\mathrm{m}} \\
\left(\mathrm{s}^{-1} \cdot \mathrm{mM}^{-1}\right)\end{array}$ & $\begin{array}{l}\text { Specific } \\
\text { actvitity } \\
\left(\mathrm{U} \cdot \mathrm{mg}^{-1}\right)\end{array}$ & $K_{\mathrm{m}}(\mathrm{mM})$ & $k_{\text {cat }}\left(\mathrm{s}^{-1}\right)$ & $\begin{array}{c}k_{\mathrm{cat}} / K_{\mathrm{m}} \\
\left(\mathrm{s}^{-1} \cdot \mathrm{mM}^{-1}\right)\end{array}$ & & \\
\hline WT & 75 & 4.5 & $905.0 \pm 11.3$ & $0.18 \pm 0.03$ & $1664.3 \pm 26.9$ & 9096.0 & $265.5 \pm 3.9$ & $10.4 \pm 0.4$ & $786.0 \pm 9.6$ & 75.8 & 1.0 & 0.29 \\
\hline F66Y & 75 & 4.5 & $892.9 \pm 19.6$ & $0.24 \pm 0.02$ & $1773.3 \pm 11.9$ & 7451.0 & $341.0 \pm 4.7$ & $4.3 \pm 0.3$ & $511.7 \pm 11.2$ & 119.0 & 1.6 & 0.38 \\
\hline E168Q & 75 & 4.5 & $858.9 \pm 12.4$ & $0.21 \pm 0.03$ & $1744.2 \pm 27.8$ & 8385.6 & $236.1 \pm 6.8$ & $5.2 \pm 0.2$ & $540.6 \pm 8.6$ & 104.0 & 1.4 & 0.27 \\
\hline M36E & 75 & 4.5 & $766.0 \pm 21.2$ & $0.25 \pm 0.03$ & $1798.0 \pm 31.6$ & 7088.0 & $256.6 \pm 4.6$ & $5.0 \pm 0.7$ & $875.4 \pm 6.9$ & 174.2 & 2.3 & 0.33 \\
\hline M36N & 75 & 4.5 & $850.8 \pm 17.1$ & $0.59 \pm 0.07$ & $1067.3 \pm 33.7$ & 1794.1 & $247.9 \pm 3.7$ & $4.8 \pm 0.4$ & $843.5 \pm 7.1$ & 177.2 & 2.3 & 0.29 \\
\hline M36G & 75 & 4.5 & $907.8 \pm 12.1$ & $1.10 \pm 0.06$ & $2566.4 \pm 15.9$ & 2320.0 & $75.2 \pm 2.8$ & $5.4 \pm 1.3$ & $115.0 \pm 3.1$ & 21.5 & 0.3 & 0.08 \\
\hline M36A & 75 & 4.5 & $876.9 \pm 26.8$ & $1.40 \pm 0.13$ & $1561.1 \pm 16.8$ & 1080.9 & $29.4 \pm 1.2$ & $5.3 \pm 0.7$ & $70.8 \pm 2.7$ & 13.3 & 0.2 & 0.03 \\
\hline M36V & 70 & 4.5 & $478.4 \pm 11.7$ & $0.80 \pm 0.04$ & $817.8 \pm 21.6$ & 1038.6 & $25.8 \pm 1.6$ & $5.2 \pm 1.2$ & $54.4 \pm 1.6$ & 10.5 & 0.1 & 0.05 \\
\hline M36L & 75 & 4.5 & $483.6 \pm 15.4$ & $0.66 \pm 0.12$ & $1279.1 \pm 14.8$ & 1932.4 & $54.5 \pm 1.7$ & $3.9 \pm 0.2$ & $89.9 \pm 3.9$ & 23.2 & 0.3 & 0.11 \\
\hline M36I & 75 & 4.5 & $390.2 \pm 11.8$ & $1.24 \pm 0.09$ & $1304.5 \pm 29.7$ & 1053.2 & $34.9 \pm 1.2$ & $4.8 \pm 0.5$ & $88.8 \pm 3.7$ & 18.4 & 0.2 & 0.09 \\
\hline M36P & 75 & 4.5 & $23.9 \pm 3.4$ & $2.10 \pm 0.13$ & $195.5 \pm 11.2$ & 93.0 & $2.9 \pm 0.3$ & $25.1 \pm 1.4$ & $22.5 \pm 1.4$ & 0.9 & 0.0 & 0.12 \\
\hline M36S & 70 & 4.5 & $32.8 \pm 2.7$ & $2.26 \pm 0.27$ & $105.8 \pm 7.9$ & 46.8 & $2.4 \pm 0.5$ & $5.7 \pm 0.4$ & $5.6 \pm 0.6$ & 1.0 & 0.0 & 0.07 \\
\hline M36C & 70 & 4.5 & $614.6 \pm 9.7$ & $0.97 \pm 0.15$ & $1888.5 \pm 51.3$ & 1956.9 & $57.8 \pm 2.9$ & $4.1 \pm 0.7$ & $116.9 \pm 6.4$ & 28.9 & 0.4 & 0.09 \\
\hline M36T & 75 & 4.5 & $593.2 \pm 13.8$ & $0.75 \pm 0.07$ & $1688.0 \pm 15.9$ & 2250.7 & $63.6 \pm 3.7$ & $5.7 \pm 1.1$ & $185.5 \pm 5.1$ & 32.7 & 0.4 & 0.11 \\
\hline M36Q & 75 & 4.5 & $408.6 \pm 10.6$ & $0.83 \pm 0.13$ & $860.4 \pm 21.8$ & 1039.8 & $27.4 \pm 0.8$ & $3.0 \pm 0.2$ & $41.0 \pm 2.8$ & 13.9 & 0.2 & 0.07 \\
\hline M36W & 75 & 4.5 & $212.6 \pm 8.7$ & $1.23 \pm 0.22$ & $1701.8 \pm 15.7$ & 1386.2 & $63.8 \pm 0.9$ & $7.3 \pm 0.2$ & $83.4 \pm 3.4$ & 11.4 & 0.2 & 0.30 \\
\hline M36Y & 75 & 4.5 & $348.6 \pm 16.9$ & $1.84 \pm 0.18$ & $2684.3 \pm 19.4$ & 1458.9 & $35.8 \pm 1.3$ & $6.1 \pm 0.1$ & $48.5 \pm 1.8$ & 7.9 & 0.1 & 0.10 \\
\hline M36F & 75 & 4.5 & $170.9 \pm 3.7$ & $1.67 \pm 0.17$ & $918.9 \pm 21.9$ & 551.3 & $24.3 \pm 0.6$ & $6.7 \pm 0.3$ & $48.5 \pm 3.1$ & 7.3 & 0.1 & 0.14 \\
\hline M36R & 70 & 4.5 & $589.1 \pm 16.9$ & $0.99 \pm 0.11$ & $1110.0 \pm 11.7$ & 1114.7 & $21.8 \pm 1.1$ & $2.5 \pm 0.2$ & $37.6 \pm 1.1$ & 14.9 & 0.2 & 0.04 \\
\hline M36H & 75 & 4.5 & $194.5 \pm 21.3$ & $1.24 \pm 0.18$ & $1382.1 \pm 10.8$ & 1114.6 & $27.8 \pm 1.1$ & $29.0 \pm 3.7$ & $737.8 \pm 21.3$ & 25.1 & 0.3 & 0.14 \\
\hline M36K & 70 & 4.5 & $208.9 \pm 19.7$ & $1.18 \pm 0.12$ & $1854.5 \pm 26.9$ & 1571.6 & $25.9 \pm 0.9$ & $27.1 \pm 2.9$ & $730.7 \pm 33.7$ & 26.7 & 0.4 & 0.12 \\
\hline M36D & 70 & 4.5 & $93.5 \pm 8.6$ & $1.94 \pm 0.07$ & $633.2 \pm 9.1$ & 325.8 & $16.5 \pm 1.7$ & $6.8 \pm 0.7$ & $31.1 \pm 2.7$ & 4.6 & 0.1 & 0.18 \\
\hline
\end{tabular}




\subsection{M36E and M36N Mutations Stabilized Trp35 Conformation by Introducing Hydrogen Bond Interactions}

To investigate the mechanism of catalytic efficiency improvement, enzyme-cellobiose complexes of dominant mutants were subjected to a $100 \mathrm{~ns}$ MD simulation analysis. The root mean square deviation (RMSD) plots of the $\alpha$-carbon atoms of five complexes plateaued during the last $80 \mathrm{~ns}$, indicating that the systems maintained equilibrium (Figure S2).

Stabilizing hydrophobic interactions between the hydrophobic stacking site Trp35 and the $\mathrm{C} 3$ atom of subsite +1 was a vitally important factor for substrate binding. Moreover, it is well known that distance was a significant determinant of the strength of hydrophobic interactions [38,39]. As for mutants M36E and M36N, the distances between the atoms Trp35@CH2/subsite+1@C3 were plotted by analyzing the dynamic trajectories and compared with wild-type Bgl3A. It was shown that the atoms Trp35@CH2/subsite +1@C3 departed from each other constantly in the wild-type, which could result in the interruption of the hydrophobic interaction, while mutants M36E and M36N exhibited more stable interaction distances (Figure 5A). Additionally, the distance values of M36E and M36N were measured to be $4.4 \AA$, and $4.1 \AA$ in the average conformations, respectively, which were much closer than the $5.7 \AA$ observed in wild-type Bgl3A (Figure 5B). Furthermore, statistics of hydrogen bond formation showed that new sets of hydrogen bonds involving the NE1 atom in the imidazole group of Trp35 were introduced in the two mutants, namely, Glu36@OE1/Trp35@NE1 or Glu36@OE2/Trp35@NE1 in M36E with a total occupancy of $38.30 \pm 1.34 \%$, and ASN36@ND2/TRP35@NE1 in M36N with an occupancy of $22.46 \pm 2.13 \%$ (Table 2). These results indicated that substitutions of the neutral and partially hydrophobic residue Met36 with the hydrophilic residue Glu36 or Asn36 gave rise to better stabilities of Trp35 by forming additional hydrogen bonds with the NE1 atom in the imidazole group, and this was in accordance with the changes of root mean square fluctuations (RMSF) of residue Trp35 (Figure S2).

Table 2. Hydrogen bond occupancy (\%) during the last $80 \mathrm{~ns}$ of the MD simulations.

\begin{tabular}{|c|c|c|c|c|c|c|c|}
\hline \multirow{2}{*}{ Acceptor } & \multirow{2}{*}{ Donor $\mathbf{H}$} & \multirow{2}{*}{ Donor } & \multicolumn{5}{|c|}{ Hydrogen Bond Occupancy $(\%)^{a}$} \\
\hline & & & WT & M36E & M36N & F66Y & E168Q \\
\hline \multicolumn{8}{|l|}{ Trp35 } \\
\hline Asn36@ND2 & Trp35@HE1 & Trp35@NE1 & $-b$ & - & $22.46 \pm 2.13 \%$ & - & - \\
\hline Glu36@OE1 & Trp35@HE1 & Trp35@NE1 & - & $38.30 \pm 1.34 \%$ & - & - & - \\
\hline \multicolumn{8}{|l|}{ Arg65 } \\
\hline Asp59@OD2 & Arg65@HH11 & Arg65@NH1 & $35.93 \pm 2.37 \%$ & $39.34 \pm 1.47 \%$ & $32.46 \pm 1.68 \%$ & $71.48 \pm 5.16 \%$ & $27.67 \pm 0.94 \%$ \\
\hline Subsite-1@O6 & Arg65@HH11 & Arg65@NH1 & $7.09 \pm 2.11 \%$ & $13.93 \pm 2.13 \%$ & $10.14 \pm 1.59 \%$ & $44.64 \pm 3.45 \%$ & $8.11 \pm 1.12 \%$ \\
\hline Ser389@OG & Arg65@HH12 & Arg65@NH1 & $45.20 \pm 4.69 \%$ & $47.08 \pm 3.18 \%$ & $39.74 \pm 2.26 \%$ & $86.03 \pm 5.34 \%$ & $43.61 \pm 3.17 \%$ \\
\hline Tyr448@OH & Arg65@HH12 & Arg65@NH1 & $79.88 \pm 3.97 \%$ & $74.71 \pm 2.26 \%$ & $79.16 \pm 4.13 \%$ & $97.85 \pm 3.89 \%$ & $86.82 \pm 5.33 \%$ \\
\hline Tyr448@OH & Arg65@HH22 & Arg65@NH2 & $12.86 \pm 1.26 \%$ & $19.25 \pm 3.14 \%$ & $18.95 \pm 2.33 \%$ & $53.11 \pm 4.11 \%$ & $15.14 \pm 2.37 \%$ \\
\hline \multicolumn{8}{|l|}{ Arg167 } \\
\hline Glu 446@OE2 & Arg167@HH22 & Arg167@NH2 & $77.88 \pm 1.98 \%$ & $79.84 \pm 6.13 \%$ & $84.84 \pm 3.11 \%$ & $73.48 \pm 2.37 \%$ & $96.13 \pm 3.51 \%$ \\
\hline Subsite +1@O6 & Arg167@HH12 & Arg167@NH1 & $29.31 \pm 1.31 \%$ & $34.44 \pm 3.17 \%$ & $30.67 \pm 3.21 \%$ & $23.25 \pm 2.15 \%$ & $49.32 \pm 4.11 \%$ \\
\hline Subsite -1@O2 & Arg167@HH22 & Arg167@NH2 & $11.17 \pm 2.18 \%$ & $13.62 \pm 2.31 \%$ & $8.26 \pm 0.91 \%$ & $15.03 \pm 1.13 \%$ & $37.53 \pm 2.34 \%$ \\
\hline
\end{tabular}

a The occupancy was the proportion of frames forming hydrogen bonds in the total frames during the MD simulation. A geometric consideration (distance of $3.5 \AA$ and an angle cutoff of $135^{\circ}$ ) was used as the criteria for hydrogen bonding. ${ }^{\mathrm{b}}$ No hydrogen bond was formed between the selected residues. 
A
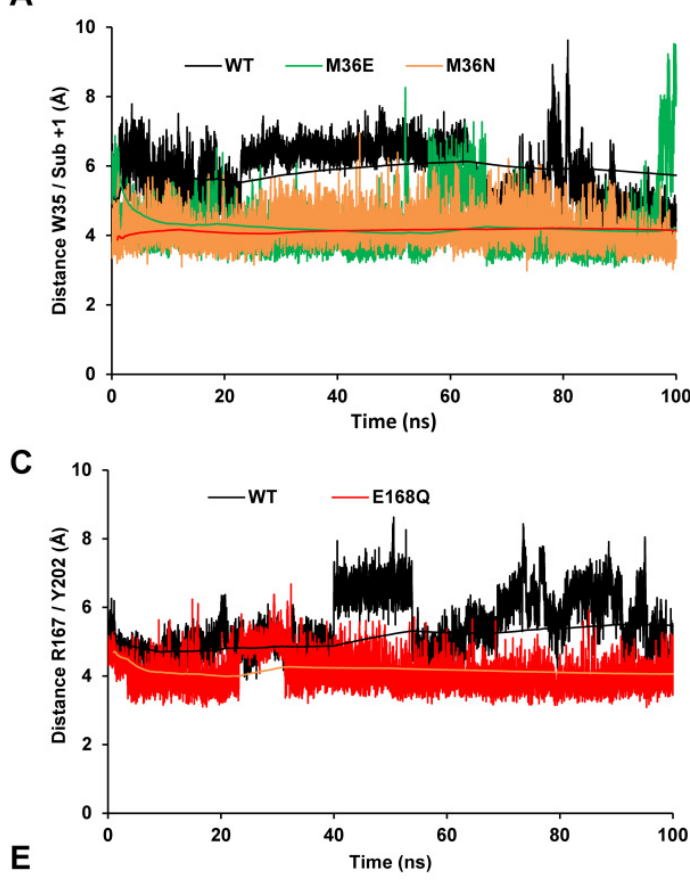

E

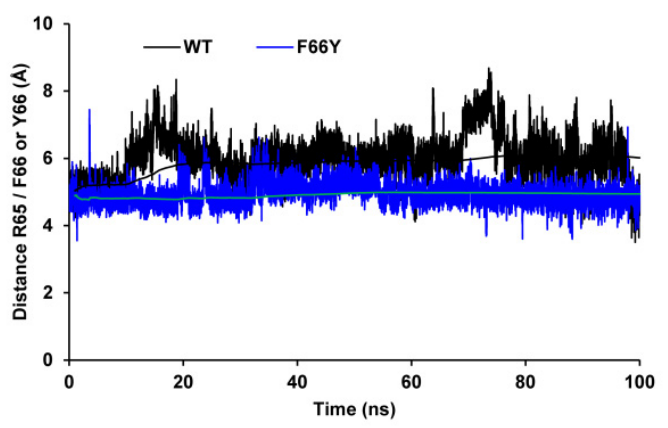

B

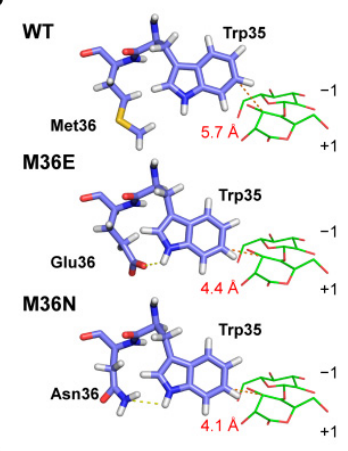

D
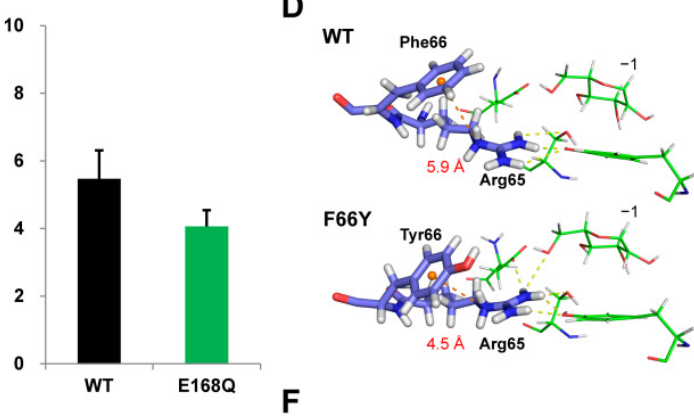

$\mathbf{F}$
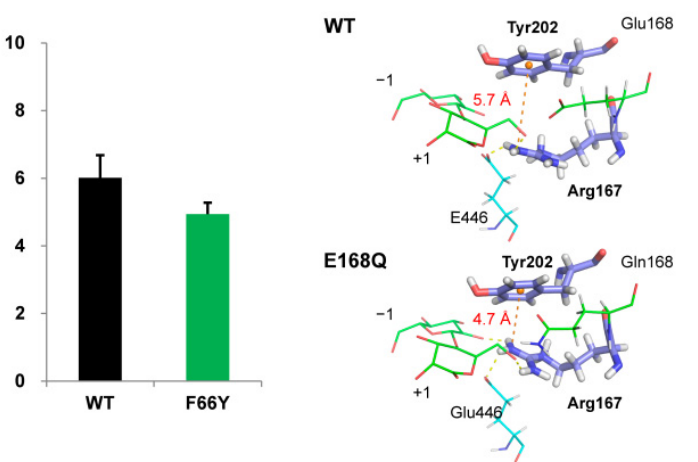

Figure 5. Distances and average conformations of investigated residues during the MD simulations. The distance between atoms Trp35@CH2 and subsite+1@C3 plotted with the simulation time (A) and the average conformation (B) in the wild-type and mutants (M36E and M36N). The distances between the positively-charged atoms of arginine and the center of mass of the benzene in cation- $\pi$ interacting residues (Arg65/Phe66 or Tyr 66 and Arg167/Tyr202) plotted along with the simulation time (C,E) and in the average conformation (D,F) in the wild-type and mutants F66Y and E168Q, respectively. Dotted yellow lines indicate the hydrogen bonds. The trend of average distance values were shown in lines and the range of distance values were plotted in bar charts.

\subsection{F66Y and E168Q Mutations Enhanced Substrate Binding by Strengthening the Cation- $\pi$ Interactions}

Figure 5C,E showed that the interval distances between the paired residues Arg65/Phe66 and Arg167/Tyr202 varied significantly in the wild-type with an average value of $5.9 \AA$ and $5.7 \AA$ during the simulation, respectively. Correspondingly, the Arg65/Phe66 distance was shortened to $4.5 \AA$ in mutant F66Y, and Arg167/Tyr202 distance was shortened to 4.7 $\AA$ in mutant E168Q (Figure 5D,F). Moreover, the migration distances of Arg65 and Arg167 in corresponding mutants decreased significantly compared with those of the wild-type, which could support more stable binding conformations. Compared with the wild-type, the amine atoms of Arg65 in mutant F66Y showed 1.99-fold, 1.90-fold, 1.63-fold, and 6.30fold hydrogen bond occupancies interacting with Asp59@OD2, Ser389@OG, Tyr448@OH, and Subsite-1@O6, respectively. Additionally, Arg167 in mutant E168Q showed 1.23fold, 1.68-fold, and 3.36-fold hydrogen bond occupancies interacting with Glu 446@OE2, Subsite+1@O6, and Subsite $-1 @ \mathrm{O} 2$, respectively (Table 2). These results suggested that 
the cation- $\pi$ interactions were strengthened in two mutants and promoted the spatial shift of Arg65 and Arg167 toward the positions facilitating the formation of hydrogen bonds with the substrate cellobiose and other polar binding sites (Figure 5D,F). The improved hydrogen bonding abilities then led to better affinities and higher catalytic efficiencies towards cellobiose. However, the increased rigidity of Arg65 and Arg167 may also be responsible for the decrease in $k_{\text {cat }}$ values.

\subsection{Binding Free Energy Calculation}

The binding free energies calculated by MM/PBSA method are shown in Table 3. It is worth noting that the lower binding free energy represented a stronger substrate affinity [40]. The mutants M36E, M36N, F66Y, and E168Q had lower binding free energies compared with the wild-type $(-22.50 \mathrm{kcal} / \mathrm{mol})$, indicating that better combinations with cellobiose were successfully achieved. Moreover, the calculated substrate affinities were ranked in the order of F66Y $>$ M36N $\geq$ M36E $>$ E168Q $>$ WT, which was in agreement with the experimentally determined $K_{\mathrm{m}}$ values. The change value of the non-polar component of solvation free energy $\Delta E_{\mathrm{np}}$ of mutants M36N and M36E were $-7.46 \mathrm{kcal} / \mathrm{mol}$ and $-9.06 \mathrm{kcal} / \mathrm{mol}$, respectively were lower than that of the wild-type and probably contributed to the improved hydrophobic interaction between the stacking site Trp35 and subsite +1 . Furthermore, the polar energy components, $\Delta E_{\mathrm{el}}$ and $\Delta E_{\mathrm{pol}}$ of the mutants F66Y and E168Q, were significantly lower than the wild-type and contributed the primary positive effect in reducing total binding free energies. Therefore, the improvement of substrate affinities of mutants F66Y and E168Q was achievable by strengthening the hydrogen bond networks centered on Arg65 and Arg167.

Table 3. Calculated energy components and binding free energies $(\mathrm{kcal} / \mathrm{mol})$ of cellobiose complexes of wild-type Bgl3A and the mutants M36E, M36N, F66Y, and E168Q.

\begin{tabular}{cccccc}
\hline Energy (kcal/mol) & WT & M36E & M36N & F66Y & E168Q \\
\hline$\Delta E_{\mathrm{vdW}}$ & $-34.84 \pm 0.39$ & $-36.48 \pm 0.70$ & $-38.17 \pm 0.49$ & $-34.72 \pm 0.99$ & $-34.29 \pm 0.78$ \\
$\Delta E_{\mathrm{el}}$ & $-85.13 \pm 0.53$ & $-83.79 \pm 1.34$ & $-83.67 \pm 0.67$ & $-89.11 \pm 0.88$ & $-90.22 \pm 1.16$ \\
$\Delta E_{\mathrm{pol}}$ & $103.07 \pm 1.75$ & $100.21 \pm 1.21$ & $100.77 \pm 1.12$ & $97.69 \pm 0.92$ & $99.40 \pm 0.83$ \\
$\Delta E_{\mathrm{np}}$ & $-5.60 \pm 0.11$ & $-7.46 \pm 0.21$ & $-9.06 \pm 0.40$ & $-7.10 \pm 0.30$ & $-5.94 \pm 0.51$ \\
\hline$G_{\text {binding }}$ & $-22.50 \pm 0.72$ & $-27.52 \pm 0.88$ & $-30.13 \pm 0.78$ & $-33.25 \pm 1.08$ & $-31.05 \pm 0.69$ \\
\hline
\end{tabular}

It is worth mentioning that the whole catalytic cycle includes three equally important processes: substrate binding, reaction, and product release. Generally, mutations in key sites of the enzyme activity center might affect all three processes simultaneously, which is finally reflected in the change of enzymatic reaction kinetic parameters. The $K_{\mathrm{m}}$ values measured experimentally were the apparent binding constants reflecting the substrate concentration requirement for the whole catalytic process of enzymes. In this study, the $K_{\mathrm{m}}$ values of the dominant mutants were significantly reduced, indicating that the substrate concentrations required by the enzymes to exert normal catalytic activities were reduced. This was direct evidence of the enzyme's improved ability to bind substrates. Besides, MD simulation and MMPBSA analysis showed that the binding energy of the mutants to the substrate was enhanced, which was consistent with the experimental results. Respect to the product, there was reason to believe that the enhanced +1 binding ability could lead to slowdown in product release. However, the distance between the two glucose molecules generated by the hydrolysis of the substrate will be larger than the distance between the two glycosylated glucosyl units before the reaction due to the break of glycosidic bonds. This meant that the +1 position of the released glucose molecule is forced to move toward the outside of the catalytic pocket, resulting in changes in binding interactions with the enzyme molecule, such as partial hydrogen bond breaking or weakening. Therefore, it could be inferred that the improvement of binding ability at +1 sites has a greater impact 
on substrate binding than on product release, which was beneficial to improve enzyme catalytic efficiency.

\section{Conclusions}

Three conserved residues (Trp36, Arg65, and Arg167) around the subsite +1 were suspected to be directly involved in the binding of glycoside substrates. Correspondingly, three adjacent residues (Met36, Phe66, and Gln168) showing variability in genetic evolution were supposed to significantly affect the conformation of the aforementioned conserved residues, therefore influencing the substrates binding. In this study, the substrate affinity and catalytic efficiency of Bgl3A were successfully improved to different degrees by engineering three residues adjacent to the conserved binding sites and indirectly taking effect, which properly conformed to our design. Consequently, the dominant mutants exhibited higher affinities and 1.4-2.3-fold catalytic efficiencies towards cellobiose. Structural and MD simulation analyses suggested that binding free energies of GH3 $\beta$-glucosidases could be obtained by either strengthened hydrophobic interactions between stacking aromatic residues and the substrate or stabilized hydrogen-bonding networks in the binding pocket. This work is expected to broaden the understanding of the substrate combination of GH3 $\beta$-glucosidases.

Supplementary Materials: The following are available online at https:/ /www.mdpi.com/article/10 $.3390 /$ biom11121882/s1, Table S1: Primers used in this study, Table S2: Specific activities and kinetic parameters of wild type Bgl3A and its mutants on cellobiose and gentiobiose, Figure S1: Structural evaluation of modeled Bgl3A by PROCHECK and Verify3D programs, Figure S2: Root mean square deviation (RMSD) and root mean square fluctuations (RMSF) plots.

Author Contributions: W.X.: conceptualization, investigation, methodology, software, visualization, writing-original draft preparation, and funding acquisition. Y.B.: investigation, formal analysis, and visualization. P.S.: conceptualization, validation, writing-review and editing, supervision, and funding acquisition. All authors have read and agreed to the published version of the manuscript.

Funding: This work was supported by the National Natural Science Foundation of China (32072166, 31801472), the Knowledge Innovation Program Funding of Institute of Food Science and Technology, Chinese Academy of Agricultural Sciences, China (125161015000150013), and the Natural Science Foundation of Jiangsu Province (BK20180604).

Institutional Review Board Statement: Not applicable.

Informed Consent Statement: Not applicable.

Data Availability Statement: Data is contained within the article or Supplementary Materials.

Conflicts of Interest: The authors declare that they have no competing interest.

\section{References}

1. Debnath, B.; Haldar, D.; Purkait, M.K. A critical review on the techniques used for the synthesis and applications of crystalline cellulose derived from agricultural wastes and forest residues. Carbohydr. Polym. 2021, 273, 118537. [CrossRef] [PubMed]

2. Tan, J.; Li, Y.; Tan, X.; Wu, H.; Li, H.; Yang, S. Advances in Pretreatment of Straw Biomass for Sugar Production. Front. Chem. 2021, 9, 1-28. [CrossRef]

3. Dadwal, A.; Sharma, S.; Satyanarayana, T. Thermostable cellulose saccharifying microbial enzymes: Characteristics, recent advances and biotechnological applications. Int. J. Biol. Macromol. 2021, 188, 226-244. [CrossRef] [PubMed]

4. Rajnish, K.N.; Samuel, M.S.; John, A.; Datta, S.; Chandrasekar, N.; Balaji, R.; Jose, S.; Selvarajan, E. Immobilization of cellulase enzymes on nano and micro-materials for breakdown of cellulose for biofuel production-A narrative review. Int. J. Biol. Macromol. 2021, 182, 1793-1802. [CrossRef]

5. Paul, M.; Mohapatra, S.; Das Mohapatra, P.K.; Thatoi, H. Microbial cellulases-An update towards its surface chemistry, genetic engineering and recovery for its biotechnological potential. Bioresour. Technol. 2021, 340, 125710. [CrossRef]

6. Bischof, R.H.; Ramoni, J.; Seiboth, B. Cellulases and beyond: The first 70 years of the enzyme producer Trichoderma reesei. Microb. Cell Factories 2016, 15, 1-13. [CrossRef]

7. Huang, Q.; Wang, K.; Li, H.; Yi, S.; Zhao, X. Enhancing cellulosic ethanol production through coevolution of multiple enzymatic characteristics of $\beta$-glucosidase from Penicillium oxalicum 16. Appl. Microbiol. Biotechnol. 2020, 104, 1-10. [CrossRef] 
8. Srivastava, N.; Rathour, R.; Jha, S.; Pandey, K.; Srivastava, M.; Thakur, V.K.; Sengar, R.S.; Gupta, V.K.; Mazumder, P.B.; Khan, A.F.; et al. Microbial Beta Glucosidase Enzymes: Recent Advances in Biomass Conversation for Biofuels Application. Biomolecules 2019, 9, 220. [CrossRef]

9. Teugjas, H.; Väljamäe, P. Selecting $\beta$-glucosidases to support cellulases in cellulose saccharification. Biotechnol. Biofuels 2013, 6, 105. [CrossRef] [PubMed]

10. Haven, M.O.; Jørgensen, H. Adsorption of $\beta$-glucosidases in two commercial preparations onto pretreated biomass and lignin. Biotechnol. Biofuels 2013, 6, 165. [CrossRef]

11. Xia, W.; Bai, Y.; Cui, Y.; Xu, X.; Qian, L.; Shi, P.; Zhang, W.; Luo, H.; Zhan, X.; Yao, B. Functional diversity of family $3 \beta$-glucosidases from thermophilic cellulolytic fungus Humicola insolens Y1. Sci. Rep. 2016, 6, 27062. [CrossRef] [PubMed]

12. Henrissat, B.; Davies, G. Structural and sequence-based classification of glycoside hydrolases. Curr. Opin. Struct. Biol. 1997, 7, 637-644. [CrossRef]

13. Lima, R.A.T.; Oliveira, G.M.; Souza, A.A.; Lopes, F.A.C.; Santana, R.H.; Istvan, P.; Quirino, B.F.; Barbosa, J.A.; Freitas, S.M.; Garay, A.V.; et al. Functional and structural characterization of a novel GH3 $\beta$-glucosidase from the gut metagenome of the Brazilian Cerrado termite Syntermes wheeleri. Int. J. Biol. Macromol. 2020, 165, 822-834. [CrossRef]

14. Xia, W.; Xu, X.; Qian, L.; Shi, P.; Bai, Y.; Luo, H.; Ma, R.; Yao, B. Engineering a highly active thermophilic $\beta$-glucosidase to enhance its $\mathrm{pH}$ stability and saccharification performance. Biotechnol. Biofuels 2016, 9, 1-12. [CrossRef] [PubMed]

15. Suzuki, K.; Sumitani, J.-I.; Nam, Y.-W.; Nishimaki, T.; Tani, S.; Wakagi, T.; Kawaguchi, T.; Fushinobu, S. Crystal structures of glycoside hydrolase family $3 \beta$-glucosidase 1 from Aspergillus aculeatus. Biochem. J. 2013, 452, 211-221. [CrossRef]

16. Karkehabadi, S.; Helmich, K.E.; Kaper, T.; Hansson, H.; Mikkelsen, N.-E.; Gudmundsson, M.; Piens, K.; Fujdala, M.; Banerjee, G.; Scott-Craig, J.S.; et al. Biochemical Characterization and Crystal Structures of a Fungal Family $3 \beta$-Glucosidase, Cel3A from Hypocrea jecorina. J. Biol. Chem. 2014, 289, 31624-31637. [CrossRef] [PubMed]

17. Pei, X.-Q.; Yi, Z.-L.; Tang, C.-G.; Wu, Z.-L. Three amino acid changes contribute markedly to the thermostability of $\beta$-glucosidase BglC from Thermobifida fusca. Bioresour. Technol. 2011, 102, 3337-3342. [CrossRef] [PubMed]

18. Marana, S.R.; Mendonça, L.M.F.; Andrade, E.H.P.; Terra, W.R.; Ferreira, C. The role of residues R97 and Y331 in modulating the $\mathrm{pH}$ optimum of an insect $\beta$-glycosidase of family 1. JBIC J. Biol. Inorg. Chem. 2003, 270, 4866-4875. [CrossRef]

19. Yadav, S.; Pandey, A.K.; Dubey, S.K. Molecular modeling, docking and simulation dynamics of $\beta$-glucosidase reveals highefficiency, thermo-stable, glucose tolerant enzyme in Paenibacillus lautus BHU3 strain. Int. J. Biol. Macromol. 2020, 168, 371-382. [CrossRef] [PubMed]

20. LaRue, K.; Melgar, M.; Martin, V.J.J. Directed evolution of a fungal $\beta$-glucosidase in Saccharomyces cerevisiae. Biotechnol. Biofuels 2016, 9, 1-15. [CrossRef]

21. Baba, Y.; Sumitani, J.-I.; Tanaka, K.; Tani, S.; Kawaguchi, T. Site-saturation mutagenesis for $\beta$-glucosidase 1 from Aspergillus aculeatus to accelerate the saccharification of alkaline-pretreated bagasse. Appl. Microbiol. Biotechnol. 2016, 100, 10495-10507. [CrossRef] [PubMed]

22. Shin, K.-C.; Hong, S.-H.; Seo, M.-J.; Oh, D.-K. An amino acid at position 512 in $\beta$-glucosidase from Clavibacter michiganensis determines the regioselectivity for hydrolyzing gypenoside XVII. Appl. Microbiol. Biotechnol. 2015, 99, 7987-7996. [CrossRef] [PubMed]

23. Sun, H.; Xue, Y.; Lin, Y. Enhanced Catalytic Efficiency in Quercetin-4'-glucoside Hydrolysis of Thermotoga maritima $\beta$-Glucosidase A by Site-Directed Mutagenesis. J. Agric. Food Chem. 2014, 62, 6763-6770. [CrossRef] [PubMed]

24. Tina, K.G.; Bhadra, R.; Srinivasan, N. PIC: Protein Interactions Calculator. Nucleic Acids Res. 2007, 35, W473-W476. [CrossRef]

25. Case, D.A.; Cheatham, T.E., III; Darden, T.; Gohlke, H.; Luo, R.; Merz, K.M., Jr.; Onufriev, A.; Simmerling, C.; Wang, B.; Woods, R.J. The Amber biomolecular simulation programs. J. Comput. Chem. 2005, 26, 1668-1688. [CrossRef]

26. Salomon-Ferrer, R.; Case, D.A.; Walker, R.C. An overview of the Amber biomolecular simulation package. Wiley Interdiscip. Rev. Comput. Mol. Sci. 2012, 3, 198-210. [CrossRef]

27. Maier, J.A.; Martinez, C.; Kasavajhala, K.; Wickstrom, L.; Hauser, K.E.; Simmerling, C. ff14SB: Improving the accuracy of protein side chain and backbone parameters from ff99SB. J. Chem. Theory Comput. 2015, 11, 3696-3713. [CrossRef]

28. Roe, D.R.; Cheatham, T.E. PTRAJ and CPPTRAJ: Software for processing and analysis of molecular dynamics trajectory data. $J$. Chem. Theory Comput. 2013, 9, 3084-3095. [CrossRef]

29. Genheden, S.; Ryde, U. The MM/PBSA and MM/GBSA methods to estimate ligand-binding affinities. Expert Opin. Drug Discov. 2015, 10, 449-461. [CrossRef]

30. Geronimo, I.; Payne, C.M.; Sandgren, M. The role of catalytic residue pKa on the hydrolysis/transglycosylation partition in family $3 \beta$-glucosidases. Org. Biomol. Chem. 2017, 16, 316-324. [CrossRef]

31. Geronimo, I.; Payne, C.M.; Sandgren, M. Hydrolysis and Transglycosylation Transition States of Glycoside Hydrolase Family 3 $\beta$-Glucosidases Differ in Charge and Puckering Conformation. J. Phys. Chem. B 2018, 122, 9452-9459. [CrossRef] [PubMed]

32. Agirre, J.; Ariza, A.; Offen, W.A.; Turkenburg, J.; Roberts, S.M.; McNicholas, S.; Harris, P.V.; McBrayer, B.; Dohnalek, J.; Cowtan, K.D.; et al. Three-dimensional structures of two heavily N-glycosylatedAspergillussp. family GH3 $\beta$-D-glucosidases. Acta Crystallogr. Sect. D Struct. Biol. 2016, 72, 254-265. [CrossRef]

33. Gudmundsson, M.; Hansson, H.; Karkehabadi, S.; Larsson, A.; Stals, I.; Kim, S.; Sunux, S.; Fujdala, M.; Larenas, E.; Kaper, T.; et al Structural and functional studies of the glycoside hydrolase family $3 \beta$-glucosidase Cel3A from the moderately thermophilic fungusRasamsonia emersonii. Acta Crystallogr. Sect. D Struct. Biol. 2016, 72, 860-870. [CrossRef] [PubMed] 
34. Karkehabadi, S.; Hansson, H.; Mikkelsen, N.E.; Kim, S.; Kaper, T.; Sandgren, M.; Gudmundsson, M. Structural studies of a glycoside hydrolase family $3 \beta$-glucosidase from the model fungusNeurospora crassa. Acta Crystallogr. Sect. F Struct. Biol. Commun. 2018, 74, 787-796. [CrossRef] [PubMed]

35. Dougherty, D.A. Cation- $\pi$ interactions in chemistry and biology: A new view of benzene, Phe, Tyr, and Trp. Science 1996, 271, 163-168. [CrossRef] [PubMed]

36. Gallivan, J.P.; Dougherty, D.A. Cation- $\pi$ interactions in structural biology. Proc. Natl. Acad. Sci. USA 1999, 17, 9459-9464. [CrossRef] [PubMed]

37. Dougherty, D.A. The Cation $-\pi$ Interaction. Acc. Chem. Res. 2012, 46, 885-893. [CrossRef]

38. Kronberg, B. The hydrophobic effect. Curr. Opin. Colloid Interface Sci. 2016, 22, 14-22. [CrossRef]

39. Privalov, P.L.; Gill, S.J. Stability of Protein Structure and Hydrophobic Interaction. Adv. Protein Chem. 1988, 39, 191-234. [CrossRef]

40. Hou, T.; Wang, J.; Li, Y.; Wang, W. Assessing the Performance of the MM/PBSA and MM/GBSA Methods. 1. The Accuracy of Binding Free Energy Calculations Based on Molecular Dynamics Simulations. J. Chem. Inf. Model. 2011, 51, 69-82. [CrossRef] [PubMed] 